\title{
Electroweak corrections and Bloch-Nordsieck violations in 2-to-2 processes at the LHC
}

\author{
W.J. Stirling, E. Vryonidou \\ Cavendish Laboratory, J.J. Thomson Avenue, Cambridge CB3 0HE, UK
}

\begin{abstract}
We consider the effect of next-to-leading order (NLO) electroweak corrections to Standard Model $2 \rightarrow 2$ processes, taking into account the potentially large double logarithms originating from both real and virtual corrections. A study of the leading Sudakov logarithms is presented and Bloch-Nordsieck $(\mathrm{BN})$ violations are discussed for processes at the CERN Large Hadron Collider. In particular, we focus on the processes $Z / \gamma+$ jet and also the ratio of $Z$ to $\gamma$ production. This ratio is known to be insensitive to NLO QCD corrections but this is not expected to be the case for the electroweak corrections. We also comment on the effect of electroweak corrections and the presence of BN violation for QCD processes, in particular dijet production, and also for purely electroweak processes such as $W+H$ and $W+Z$ associated production.
\end{abstract}




\section{Contents}

1. Introduction 1

2. Sudakov Double Logs in real and virtual corrections 3

3. $\gamma / Z$ plus jet production

3.1 Partonic results 1

3.1.1 Initial state: $q \bar{q}$

3.1.2 Initial state: $q g$

3.1.3 Isospin averaging - cancellation of double logarithms

3.2 Hadronic results 8

3.2.1 Comparison with numerical calculation of full real corrections 9

3.2.2 The $\gamma$ to $Z$ ratio 10

4. Bloch-Nordsieck violations in other hard-scattering processes 12

4.1 QCD dijet production $\quad 12$

4.2 Electroweak processes

4.2.1 $\mathrm{W}+\mathrm{Z}$ production

4.2.2 $\mathrm{W}+\mathrm{H}$ production 16

5. Conclusions 17

\section{Introduction}

With the LHC in full operation, the experiments are collecting enormous amounts of data that are then used to obtain precision measurements of various observables. These measurements are used to search for New Physics and also to test the Standard Model (SM). In order to maximize the information that can be extracted in this way, the uncertainty in the theoretical predictions needs to be reduced. This has motivated the calculation of many processes at NLO in perturbative QCD and in some cases at NNLO QCD. In addition to QCD corrections, electroweak corrections also need to be taken into account. At centre-ofmass energies of a few $\mathrm{TeV}$ these can become numerically important, since in many cases they are known to take the form of Sudakov-like logarithms $\sim \alpha_{w} \log ^{2}\left(\mu^{2} / M_{W}^{2}\right)$, with $\mu$ a typical hard scale in the process. This has been known for a long time, and consequently electroweak corrections have been considered for a wide range of hard-scattering processes.

Virtual electroweak (EW) corrections have been calculated for a range of SM processes such as $Z / \gamma+$ jet [1-3], inclusive jet production [4], top pair production [5,6], and b-jet production [7]. Previous work has generally focused on the virtual EW corrections. Real 
corrections are usually not taken into account, even though they contribute at the same order in perturbation theory. This approach can be justified by arguing that real emission of $W$ and $Z$ bosons, which subsequently decay, leads to qualitatively different final states with different (and more complex) signatures in the detectors. Moreover as the $W$ and $Z$ masses act as infrared regulators, EW virtual corrections remain finite, in contrast to QCD corrections where the addition of real corrections is necessary to cancel the soft and collinear divergences. However, exclusion of the real emission contribution is only justified when exclusive final states are considered. In contrast, when fully inclusive final states are studied, the effect of virtual corrections is expected to be at least partially compensated by the effect of real electroweak gauge boson emission. Nevertheless, and as we shall discuss in detail below, the cancellation is not expected to be exact due to violation of the Bloch-Nordsieck (BN) theorem even for fully inclusive observables, see for example [8-11]. The BN violation originates from the non-abelian nature of the electroweak charges in the initial partonic state and is related to electroweak symmetry breaking. The same violation is expected in QCD, as QCD is also a non-abelian theory. However in this case it has no physical consequences because of the colour averaging of the initial partonic states. In the electroweak case the initial-state weak isospin charge is fixed and there is no averaging, which leads to non-cancelling Sudakov logarithms of the form $\sim \alpha_{w} \log ^{2} \frac{s}{M_{V}^{2}}$, with $V=W, Z$. Averaging over different possible weak isospin initial states (e.g. $\sigma_{e \bar{e}}+\sigma_{\nu \bar{e}}$ or $\left.\sigma_{u g}+\sigma_{d g}\right)$ does lead to a full cancellation of the BN violating Sudakov logs. In practice, for hadron colliders this averaging is not possible as different weak isospin (quark and gluon) states receive different parton distribution function (PDF) weights. As discussed in [9], $\mathrm{BN}$ violating terms only originate from initial state radiation, with final state radiation logarithms cancelling between virtual and real corrections. Moreover, the BN violating logs are related only to $W$ boson emission. Sudakov $\operatorname{logs}$ from $\gamma / Z$ emission cancel between real and virtual corrections, as $\gamma / Z$ emission does not change the particle type.

The combined effect of real and one-loop virtual corrections for $Z$ and $\gamma$ production, as well as for other SM processes such as inclusive jet and Drell-Yan production, has been considered in [12], where a partial cancellation is found. The effect of selection cuts on the quantitative importance of BN violating Sudakov logs has been considered in [13]. Even though the analysis focuses on the simpler case of an $e^{-} e^{+}$collider, the general conclusion drawn is that the extent of the compensation between the large (negative) logs of the virtual corrections and the large (positive) logs associated with the real emission depends strongly on the phase space cuts applied to the real radiation, i.e. how much of the collinear and soft $W$ and $Z$ radiation is allowed to escape detection.

In this paper we study how the real emission of soft EW bosons compensates the large negative Double Logarithms (DL) associated with the virtual corrections in hard scattering processes at the LHC. In Section 2 we review the emergence of Sudakov logs in virtual and real corrections and the origin of $\mathrm{BN}$ violations. In Section 3 we obtain the real corrections for $\gamma / Z+1$ jet production at the LHC and combine them with the virtual logs. In Section 4, we comment on BN violation in other $2 \rightarrow 2$ scattering processes such as QCD dijet production and pure electroweak processes, before we conclude in Section 5 . 


\section{Sudakov Double Logs in real and virtual corrections}

In this section we give a brief overview of Sudakov logs in EW corrections. In order to do this at the DL level we need a recipe for the extraction of the DL for both virtual and real corrections. For the real corrections this is achieved by inserting the eikonal current for the emission of a soft gauge boson of momentum $k$ :

$$
J^{\mu}(k)=g_{w} \sum_{i=1}^{n} T_{i} \frac{p_{i}^{\mu}}{p_{i} k}
$$

with $T_{i}$ the relevant isospin generator and the sum running over all external legs. Squaring the eikonal current and integrating over the phase space of the emitted gauge boson, we obtain a factor multiplying the LO result:

$$
\int_{M}^{E} \frac{d^{3} k}{2 \omega_{k}(2 \pi)^{3}} \frac{2 p_{i} p_{j}}{\left(k p_{i}\right)\left(k p_{j}\right)}=\frac{1}{8 \pi^{2}} \log ^{2} \frac{2 p_{i} p_{j}}{M^{2}},
$$

in the high-energy limit of $M^{2} \ll 2 p_{i} p_{j} \sim E^{2}$, leading to a DL of the form

$$
I(k)=\frac{\alpha_{w}}{4 \pi} \sum_{i<j} T_{i} \cdot T_{j} \log ^{2} \frac{2 p_{i} p_{j}}{M^{2}}
$$

with $i$ and $j$ external legs and $\alpha_{w}=g_{w}^{2} / 4 \pi$ with the contribution being non-vanishing only when $T_{i} \cdot T_{j}$ is non-zero. In a Feynman diagram interpretation, for the real corrections it is the interference between two diagrams where the gauge boson is emitted from two different external legs that leads to the DL. In practice, the correction takes the schematic factorised form: $\frac{\alpha_{w}}{4 \pi} \mathrm{L}^{2}\left|M_{L O}\right|^{2}$ with $\mathrm{L}=\log ^{2}\left(2 p_{i} p_{j} / M^{2}\right)$, and an appropriate prefactor determined by the couplings.

For the virtual corrections a generalised method for extracting soft and collinear logs in the high-energy limit was presented in [14]. The DL originate from the exchange of soft gauge bosons only between external legs. The method can be used for any process and the change in the matrix element due to the loop correction is given by

$$
\delta M^{i_{1} \ldots i_{n}}=\frac{1}{2} \sum_{k=1}^{n} \sum_{l \neq k} \sum_{V_{a}=A, Z, W^{ \pm}} I_{i_{k}^{\prime} i_{k}}^{V_{a}}(k) I_{i_{l}^{\prime} i_{l}}^{\bar{V}_{a}}(l) M_{0}^{i_{1} \ldots i_{k}^{\prime} \ldots i_{l}^{\prime} \ldots i_{n}} \frac{\alpha_{w}}{4 \pi} \log ^{2} \frac{2 p_{k} p_{l}}{M_{V_{a}}^{2}},
$$

for a process with $n$ external legs and tree-level LO matrix element $M_{0}^{i_{1} \ldots i_{n}}$, and $I_{i_{k}^{\prime} i_{k}}^{V_{a}}(k)$ the $\mathrm{SU}(2) \times \mathrm{U}(1)$ generators with $i e I_{i_{k}^{\prime} i_{k}}^{V_{a}}(k)$ giving the coupling for the $V_{a} \bar{\phi}_{i} \phi_{i}^{\prime}$ vertex, e.g. $I_{e^{+} e^{-}}^{\gamma}=1$. This expression can be further simplified under certain assumptions leading to a single sum over the external legs as shown in [14]:

$$
\delta M^{i_{1} \ldots i_{n}}=\sum_{k=1}^{n}-\frac{1}{2} C_{i_{k}^{\prime} i_{k}}^{e w} M_{0}^{i_{1} \ldots i_{k}^{\prime} \ldots i_{n}} \frac{\alpha_{w}}{4 \pi} \log ^{2} \frac{s}{M_{W}^{2}},
$$

with $C^{e w}$ the Casimir operator as defined in [14] and where all logarithms of pure electromagnetic origin or of the form $\log \left(M_{Z}^{2} / M_{W}^{2}\right)$ are neglected. In practice, we keep the 
first form given in Eq. (2.4) to facilitate the comparison with the real corrections. The generator prefactors match between the corresponding combinations of external legs in real and virtual corrections. For a fixed initial state the extent of the BN violation depends on which real emission processes are allowed, as $W^{ \pm}$emission might be forbidden due to charge conservation. When both emission from external leg $k$-absorption from leg $l$ (virtual) and interference between the two emissions (real) are allowed, it is the difference between $M_{0}^{i_{1} \ldots i_{k}^{\prime} \ldots i_{l}^{\prime} \ldots i_{n}} M_{0}^{i_{1} \ldots i_{k} \ldots i_{l} \ldots i_{n}}$ and $M_{0}^{i_{1} \ldots i_{k}^{\prime} \ldots i_{l} \ldots i_{n}} M_{0}^{i_{1} \ldots i_{k} \ldots i_{l}^{\prime} \ldots i_{n}}$ that determines whether the $\mathrm{BN}$ theorem is violated. In the case of neutral gauge bosons $(\gamma, Z)$, the two expressions are always the same as the particle type does not change, i.e. $i_{k}^{\prime}=i_{k}$. This explains more formally the observation that only $W$ boson emission leads to $\mathrm{BN}$ violating logs.

Using Eq. (2.4) we can identify the origin of the DL in the virtual corrections discussed in the following sections, with the prefactors obtained from the corresponding couplings between the gauge bosons and the emitting external particles. The interference of $\delta M^{i_{1} \ldots i_{n}}$ with the LO diagram gives the contribution of the virtual corrections to the matrix element squared:

$$
\delta\left|M^{i_{1} \ldots i_{n}}\right|^{2}=\sum_{k=1}^{n} \sum_{l \neq k} \sum_{V_{a}=A, Z, W^{ \pm}} I_{i_{k}^{\prime} i_{k}}^{V_{a}}(k) I_{i_{l}^{\prime} i_{l}}^{\bar{V}_{a}}(l) M_{0}^{i_{1} \ldots i_{k}^{\prime} \ldots i_{l}^{\prime} \ldots i_{n}} M_{0}^{* i_{1} \ldots i_{n}} \frac{\alpha_{w}}{4 \pi} \log ^{2} \frac{2 p_{k} p_{l}}{M_{V_{a}}^{2}},
$$

while the corresponding result for the real corrections takes the slightly different form:

$$
\delta\left|M^{i_{1} \ldots i_{n}}\right|^{2}=-\sum_{k=1}^{n} \sum_{l \neq k} \sum_{V_{a}=A, Z, W^{ \pm}} I_{i_{k}^{\prime} i_{k}}^{V_{a}}(k) I_{i_{l}^{\prime} i_{l}}^{\bar{V}_{a}}(l) M_{0}^{i_{1} \ldots i_{k}^{\prime} \ldots i_{n}} M_{0}^{* i_{1} \ldots i_{l}^{\prime} \ldots i_{n}} \frac{\alpha_{w}}{4 \pi} \log ^{2} \frac{2 p_{k} p_{l}}{M_{V_{a}}^{2}}
$$

These two expressions can be used to check whether a process will exhibit BN violations. We will use them for specific processes in the following sections.

\section{3. $\gamma / Z$ plus jet production}

\subsection{Partonic results}

We start our study by focusing on $\gamma / Z+1$ jet production, for which analytic results in the high-energy limit for the virtual corrections are already available in the literature [1-3]. We will combine these with our real correction calculations. In this calculation $\gamma$ and $Z$ are produced on-shell and no decays are taken into account. Also no pure electromagnetic corrections will be taken into account. We start by considering $\gamma+1$ jet production as an example. The virtual correction diagrams are shown in [3]. As discussed there, the virtual corrections in the high-energy limit $\left(s, t, u \gg M_{Z / W}^{2}\right)$ take the form of Sudakov logarithms, $\log \left(r / M_{V}^{2}\right)$ where $M_{V}$ is the mass of the exchanged boson and $r$ one of the Mandelstam variables $s, t, u$, following Eq. (2.4). The virtual corrections are extracted in the approximation $M_{Z}=M_{W}$. To obtain the corresponding leading logs from the real corrections, we work in the same limit with the eikonal current. For fixed initial state $q \bar{q}$ the real boson emitted is always a $Z$, to conserve charge, while for the $q g$ initial state the emitted electroweak boson can be either a $Z$ or $W$, with $W$ changing the flavour of the final state quark. In the following subsections for illustration purposes we present some analytic 
partonic results for a selection of initial states, to examine the $\mathrm{BN}$ violating effects, while hadronic numerical results will be presented in a subsequent subsection.

\subsubsection{Initial state: $q \bar{q}$}

We start by considering as an example $q\left(p_{1}\right) \bar{q}\left(p_{2}\right) \rightarrow \gamma\left(p_{3}\right) g\left(p_{4}\right)$. The one-loop virtual corrections are calculated in [3] to give the matrix element squared:

$$
\bar{\sum}\left|M^{q \bar{q}}\right|^{2}=8 \pi^{2} \alpha_{w} \alpha_{s}\left(N_{c}^{2}-1\right) \frac{\hat{t}^{2}+\hat{u}^{2}}{\hat{t} \hat{u}}\left[2 Q_{q}^{2}+\frac{\alpha_{w}}{2 \pi} A^{(1)}\right]
$$

with $A^{(1)}$ given in the high-energy limit by

$$
A^{(1)}=-\sum_{\lambda=L, R} Q_{q}\left[Q_{q}\left(C_{q \lambda}^{e w}-Q_{q}^{2}\right)\left(L_{s}^{2}-3 L_{s}\right)+\frac{1}{s_{w}^{2}} T_{q \lambda}^{3}\left(L_{t}^{2}+L_{u}^{2}-L_{s}^{2}\right)\right]
$$

with $s=2 p_{1} p_{2}, t=-2 p_{1} p_{3}, u=-2 p_{2} p_{3}, L_{r}^{k}=\log ^{k}\left(|r| / M_{W}^{2}\right), Q_{q}$ the charge, $T_{q}^{3}$ the isospin generator, $C^{e w}$ the Casimir operator and $s_{w}=\sin \theta_{w}$. The second term in $A^{(1)}$ comes from the exchange of a virtual $W$ boson and is therefore non-zero only for left-handed quarks, while the first is related to both $W$ and $Z$ exchange. The two contributions can be disentangled as $\left(C_{q \lambda}^{e w}-Q_{q}^{2}\right)=\sum_{Z, W^{ \pm}}\left(I_{V} I_{\bar{V}}\right)_{q_{\lambda}}$. As an example, we show the $A^{(1)}$ results for $u \bar{u}$, substituting the corresponding couplings:

$$
A^{(1)}=-\frac{2}{3}\left[\frac{2}{3}\left(\left(\left(\frac{c_{w}}{2 s_{w}}-\frac{s_{w}}{6 c_{w}}\right)^{2}+\frac{1}{2 s_{w}^{2}}\right)+\frac{4 s_{w}^{2}}{9 c_{w}^{2}}\right)\left(L_{s}^{2}-3 L_{s}\right)+\frac{1}{2 s_{w}^{2}}\left(L_{t}^{2}+L_{u}^{2}-L_{s}^{2}\right)\right]
$$

with $c_{w}=\cos \theta_{w}$. The virtual correction can be written as

$$
\delta^{v} \bar{\sum}\left|M^{u \bar{u}}\right|^{2}=-\frac{\alpha_{w}}{144 \pi c_{w}^{2} s_{w}^{2}}\left[\left(9-24 s_{w}^{2}+32 s_{w}^{4}\right) L_{s}^{2}-9 c_{w}^{2} L_{s}^{2}+27 c_{w}^{2}\left(L_{t}^{2}+L_{u}^{2}\right)\right] \bar{\sum}\left|M_{L O}^{u \bar{u}}\right|^{2},
$$

with the first bracket coming from virtual $Z$ exchange and the rest from $W$ exchange.

The real correction contributions are obtained from the relevant Feynman diagrams. In this case, because of charge conservation the only allowed emission is that of a $Z$ boson as shown in Fig. 1. Taking the appropriate limit of soft $p_{5}$, the $2 \rightarrow 3$ correction to the matrix element squared factorises into

$$
\delta^{r} \bar{\sum}\left|M^{u \bar{u}}\right|^{2}=\bar{\sum}\left|M_{L O}^{u \bar{u}}\right|^{2} \frac{\pi \alpha_{w}}{18 c_{w}^{2} s_{w}^{2}}\left(9-24 s_{w}^{2}+32 s_{w}^{4}\right) \frac{2 p_{1} p_{2}}{\left(p_{1} p_{5}\right)\left(p_{2} p_{5}\right)}
$$

and integrating over $p_{5}$

$$
\rightarrow \frac{\alpha_{w}}{144 \pi c_{w}^{2} s_{w}^{2}}\left(9-24 s_{w}^{2}+32 s_{w}^{4}\right) L_{s}^{2} \bar{\sum}\left|M_{L O}^{u \bar{u}}\right|^{2},
$$

with $p_{1}$ and $p_{2}$ the quark and antiquark momenta respectively. Here we can identify the eikonal factor of Eq. (2.2) which leads to a double logarithm of the form $\log ^{2}\left(s / M_{Z}^{2}\right)$ when integrated over the phase space of the soft $Z$. The $s$-log is expected from the Feynman 


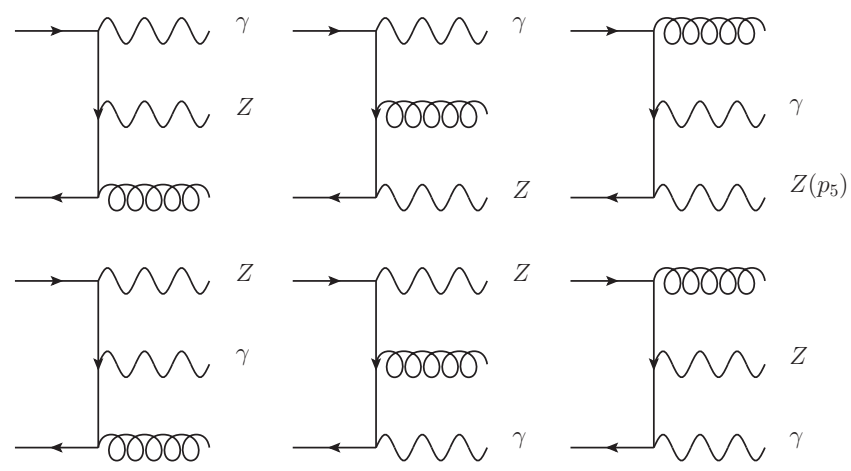

Figure 1: Feynman diagrams for $u\left(p_{1}\right) \bar{u}\left(p_{2}\right) \rightarrow A\left(p_{3}\right) g\left(p_{4}\right) Z\left(p_{5}\right)$.

diagrams, as the emission of the soft $Z$ can occur from the both initial state legs and the logarithm arises from the interference of the two corresponding diagrams. The polynomial in $\sin \theta_{w}$ prefactor, $9-24 s_{w}^{2}+32 s_{w}^{4}$, is determined by the $u$-quark couplings to the $Z$ and is in fact proportional to $c_{A}^{2}+c_{V}^{2}$. For the $d \bar{d}$ initial state this would change to $9-12 s_{w}^{2}+8 s_{w}^{4}$. It is clear from Eqs. (3.3) and (3.6) that the cancellation between real and virtual corrections logarithms is not exact. Virtual corrections include logarithms of all $s, t$ and $u$. By checking the DL term by term, we can verify that the logarithms related to virtual soft $Z$ exchange - the first and third terms of the first bracket in Eq. (3.3) - are exactly cancelled by the real corrections. The remaining terms are due to the exchange of virtual $W$ bosons and these cannot be cancelled simply due to the absence of real $W$ emission diagrams.

\subsubsection{Initial state: $q g$}
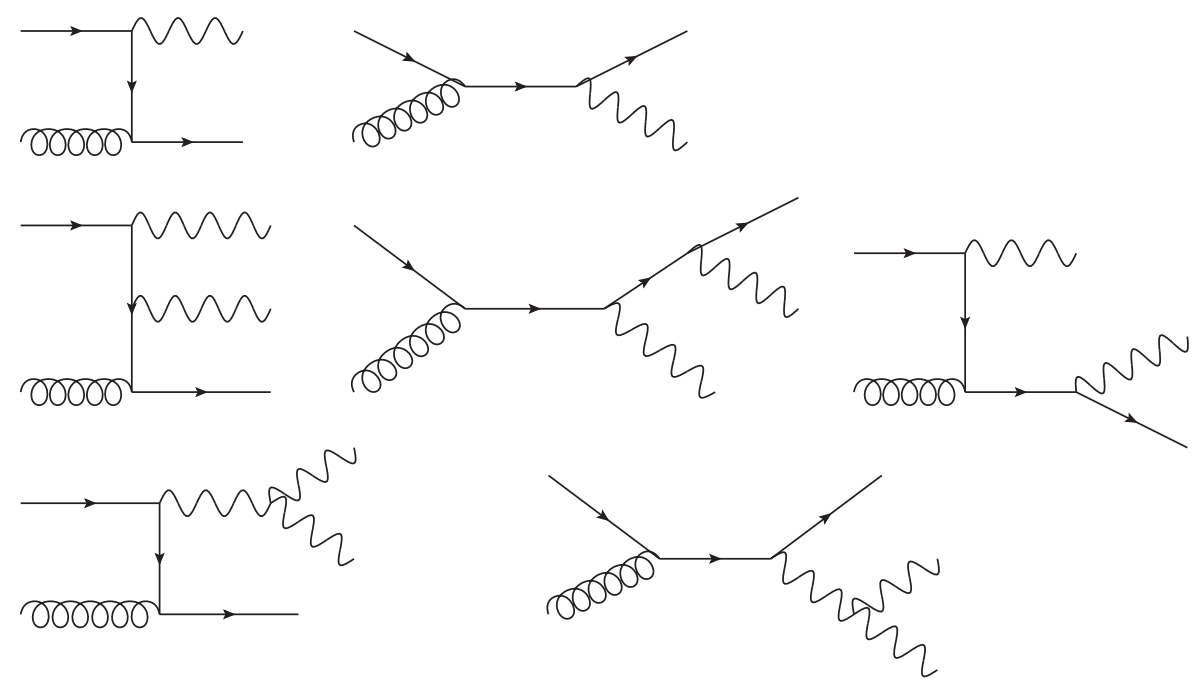

Figure 2: Feynman diagrams for $q g \rightarrow \gamma q$.

The topology of the relevant Feynman diagrams for initial-state $q g$, which is found 
to be the dominant process for $\gamma+1$ jet production at the LHC, is shown in Fig. 2 with the LO diagrams in the first row and the (NLO) real emission diagrams in the second and third rows, where an additional electroweak gauge boson is emitted. The topology of the last two diagrams only applies for $W$ emission. In the case of $u g \rightarrow \gamma u$ the real EW corrections include both $u g \rightarrow \gamma u Z$ and $u g \rightarrow \gamma d W^{+}$. Calculating the matrix element for $u\left(p_{1}\right) g\left(p_{2}\right) \rightarrow \gamma\left(p_{3}\right) u\left(p_{4}\right) Z\left(p_{5}\right)$ we obtain

$$
\delta^{r} \bar{\sum}\left|M^{u g}\right|^{2}=\bar{\sum}\left|M_{L O}^{u g}\right|^{2} \frac{\alpha_{w}}{144 \pi c_{w}^{2} s_{w}^{2}}\left(9-24 s_{w}^{2}+32 s_{w}^{4}\right) \log ^{2} \frac{2 p_{1} p_{3}}{M_{Z}^{2}} .
$$

Note that the prefactor $\left(9-24 s_{w}^{2}+32 s_{w}^{4}\right)$ is the same as for the $u \bar{u}$ initial state. The result can be obtained from Eq. (3.6) by appropriate use of crossing relations and modification of the colour averaging factor at LO.

For the $u\left(p_{1}\right) g\left(p_{2}\right) \rightarrow d\left(p_{3}\right) \gamma\left(p_{4}\right) W^{+}(p 5)$ process we obtain

$$
\delta^{r} \bar{\sum}\left|M^{u g}\right|^{2}=\bar{\sum}\left|M_{L O}^{u g}\right|^{2} \frac{\alpha_{w}}{32 s_{w}^{2} \pi}\left[3 L_{u}^{2}-2 L_{t}^{2}+6 L_{s}^{2}\right],
$$

in the soft $W$ limit. The prefactor of each of the logarithms is determined by the couplings/charge of the quark involved. In a similar way results for all the other processes, e.g. $u \bar{d} \rightarrow \gamma g W^{+}$, can be obtained by crossing relations. The results for the virtual corrections can be obtained from Eq. (3.1) by making the substitutions: $s \rightarrow u=-2 p_{1} p_{3}, t \rightarrow$ $s=2 p_{1} p_{2}=2 p_{3} p_{4}, u \rightarrow t=-2 p_{1} p_{4}$ in the high-energy limit, giving for the leading-log (LL) result:

$$
\delta^{v} \bar{\sum}\left|M^{u g}\right|^{2}=-\frac{\alpha_{w}}{144 \pi c_{w}^{2} s_{w}^{2}}\left[\left(9-24 s_{w}^{2}+32 s_{w}^{4}\right) L_{u}^{2}-9 c_{w}^{2} L_{u}^{2}+27 c_{w}^{2}\left(L_{s}^{2}+L_{t}^{2}\right)\right] \bar{\sum}\left|M_{L O}^{u g}\right|^{2} .
$$

By comparing Eq. (3.7) to Eq. (3.9) we again see that the logarithms due to $Z$ exchange cancel, with the appropriate decomposition to $W$ and $Z$ contributions. Furthermore the logarithms related to final state radiation, i.e. the term proportional to $\log ^{2}\left(\frac{2 p_{3} p_{4}}{M_{W}^{2}}\right)=$ $\log ^{2}\left(\frac{s}{M_{W}^{2}}\right)$ in Eq. (3.8), exactly cancels the equivalent term which appears in Eq. (3.9). This is a good demonstration of the conclusions reached in [9], regarding the difference between $W$ and $Z$ emission and also the final state radiation logs.

\subsubsection{Isospin averaging - cancellation of double logarithms}

Another important check for the analytic calculations is the cancellation of the logarithms on averaging over two states of the same weak isospin doublet. As an example, we take the results for $u g \rightarrow \gamma u$ and $d g \rightarrow \gamma d$ with their corresponding virtual and real corrections. Even though for the two processes separately the sum of real and virtual corrections is BN violating, when we sum the two with equal weights the logarithms cancel. This can be seen explicitly by considering the corresponding results for $d g \rightarrow \gamma d$ :

$$
\delta^{v} \bar{\sum}\left|M^{d g}\right|^{2}=-\frac{\alpha_{w}}{144 \pi c_{w}^{2} s_{w}^{2}}\left[\left(9-12 s_{w}^{2}+8 s_{w}^{4}\right) L_{u}^{2}-36 c_{w}^{2} L_{u}^{2}+54 c_{w}^{2}\left(L_{s}^{2}+L_{t}^{2}\right)\right] \bar{\sum}\left|M_{L O}^{d g}\right|^{2},
$$




$$
\begin{gathered}
\delta^{r} \bar{\sum}\left|M^{d g}\right|^{2}=\bar{\sum}\left|M_{L O}^{d g}\right|^{2} \frac{\alpha_{w}}{144 \pi c_{w}^{2} s_{w}^{2}}\left(9-12 s_{w}^{2}+8 s_{w}^{4}\right) L_{u}^{2}, \\
\delta^{r} \bar{\sum}\left|M^{d g}\right|^{2}=\bar{\sum}\left|M_{L O}^{d g}\right|^{2} \frac{\alpha_{w}}{8 s_{w}^{2} \pi}\left[3 L_{s}^{2}-2 L_{t}^{2}+6 L_{u}^{2}\right] .
\end{gathered}
$$

Adding up all relevant expressions for $u g$ (Eqs. (3.7), (3.8) and (3.9)) and $d g$ (Eqs. (3.10), (3.11) and (3.12)) and taking into account the factor of 4 difference between $\left|M_{L O}^{d g}\right|^{2}$ and $\left|M_{L O}^{u g}\right|^{2}$, due to electric charge, we find that no Sudakov logs remain uncancelled. Of course in practice this averaging is not possible, as in hadronic collisions $u$ - and $d$-quark contributions are weighted by the corresponding $u$ - and $d$-quark PDFs which are of course different. The importance of the $\mathrm{BN}$ violating terms is then determined by the values of the PDF momentum fractions and factorisation scales that dictate the relative size of the PDF values, and therefore change the relative cancellation between different flavours.

\subsection{Hadronic results}

$Z+1$ jet production can be treated in a similar way. Partonic results have been extracted and shown to lead to the same conclusions on BN violations, but we do not show the expressions here as these are more lengthy due to the more complex structure of the $Z$ coupling to quarks. The partonic results can then be used to obtain hadronic cross sections by convoluting with the appropriate PDFs. For consistency we note that as we have not included any single logs (SL) from the real corrections, we only keep the DL in the expressions for the virtual corrections and therefore our results are valid only at the LL level. However we do need to keep in mind that the SL contribution remains important even at very high $p_{T}$. This is shown in Fig. 3, where the fraction of the virtual corrections to $\gamma+1$ jet production coming from the SL terms is shown for the LHC (at $14 \mathrm{TeV}$ ) as a function of the photon $p_{T}$, with no cuts applied. We see that the next-to-leading logarithms remain important even at high transverse momentum (at $2 \mathrm{TeV}$ the contribution is still more than $20 \%$ ).

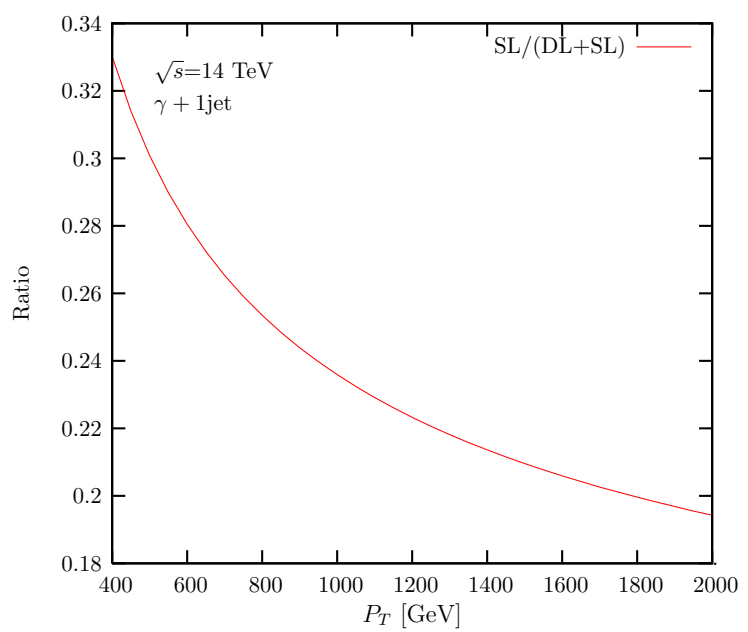

Figure 3: Relative contribution of single logarithms to the virtual corrections in $\gamma+1$ jet production. 
The results for the $p_{T}^{\gamma}$ differential distribution for $\gamma+1$ jet production at the LHC, with only the virtual corrections of [3] and then with the addition of the real corrections are shown in Fig. 4 , with $R_{L L / L O}=L L / L O-1$. These are obtained using MSTW2008LO PDFs [15] and setting the factorisation and renormalisation scales to $p_{T}^{\gamma}$. We note that the virtual corrections decrease the cross section by up to $20 \%$ at very high $p_{T}(2 \mathrm{TeV})$ due to the large negative Sudakov logs. We note that no phase space constraints are imposed on the emitted gauge bosons other than the eikonal current approximation used to extract the leading logarithms. This computational set-up does not allow the application of cuts on the emitted gauge bosons or their decay products as we work in a two-body phase space approximation, with the LO result multiplied by a factor of the form $\left(1+C \alpha_{w} \log ^{2}\left(r / M^{2}\right)\right)$. We note that adding the real correction due to $W$ emission has a bigger effect than that due to $Z$ emission. This is a combination of the different quark couplings to $W$ and $Z$ and the fact that including the $\mathcal{O}\left(\alpha_{W}^{2}\right)$ real $W$ corrections involves including new initial-state parton combinations e.g. $u \bar{d}, d \bar{u}$ etc., which are not allowed at LO and these increase the cross section. The calculation is performed using a diagonal CKM matrix. Evidently there is a significant but not exact compensation of the negative virtual contribution by the real corrections at the DL level.

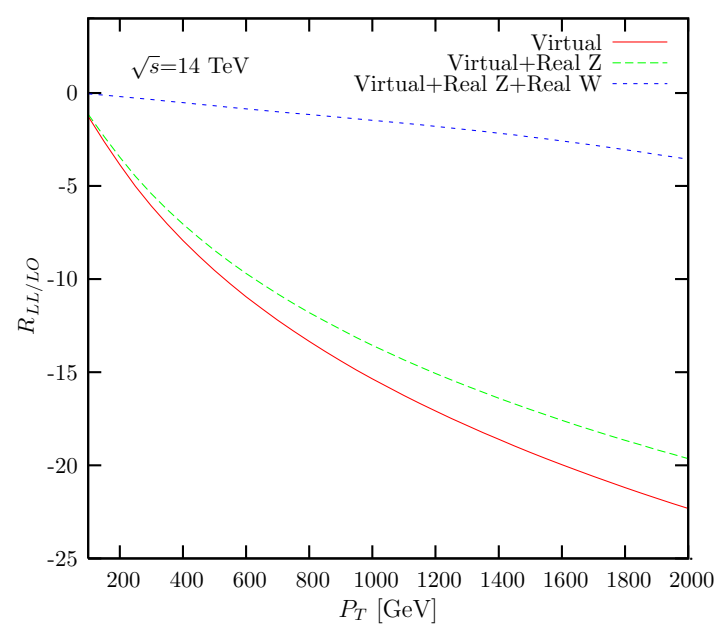

Figure 4: Virtual and real electroweak corrections to $\gamma+1$ jet production at $14 \mathrm{TeV}$.

The same procedure is followed for $Z+1$ jet, with the analytic DL results of the partonic cross sections convoluted with the PDFs. In the calculation of the matrix elements for the partonic cross sections, we ignore the mass of the $Z$. This is a good approximation in the region of high $p_{T}$ where the LL approximation is most valid. The results are shown in Fig. 5. In this case it can be seen than the real corrections overcompensate the negative double logarithms of the virtual corrections, with the $W^{ \pm}$contribution being dominant.

\subsubsection{Comparison with numerical calculation of full real corrections}

Our results for the real corrections have been obtained using the eikonal current to extract the leading logarithmic terms. This can be compared to the full real corrections result obtained using the exact matrix elements for the $2 \rightarrow 3$ processes and therefore a 3 -body 


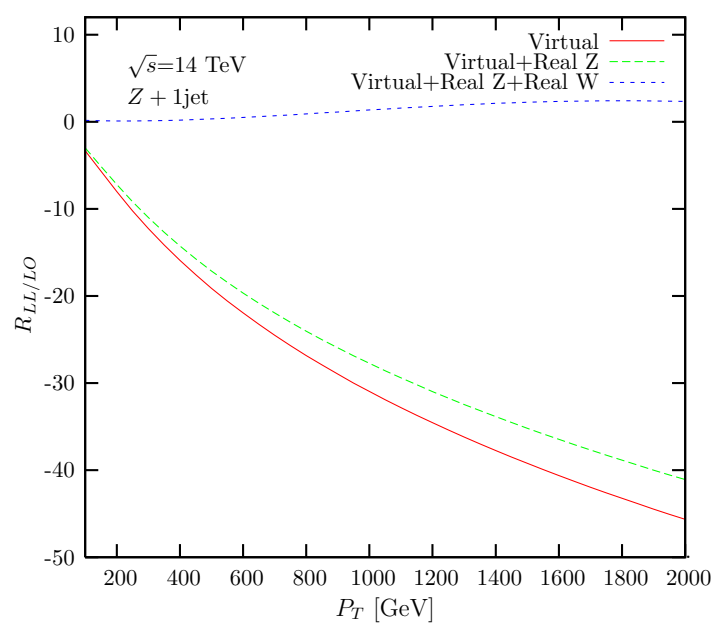

Figure 5: Virtual and real electroweak corrections to $Z+1$ jet production at $14 \mathrm{TeV}$.

phase space. This can be done using publicly available programmes such as MCFM [16]. This combination of the real and virtual corrections has been first discussed in [12] for a series of SM processes, including $\gamma / Z+$ jet production.

Here we select two specific processes, corresponding to initial states $u \bar{u}$ and $u g$, to gauge the differences in the two approaches. We use the same PDFs, scale and EW parameters. The results are shown in Figs. 6 and 0, where we show the photon $p_{T}$ distribution for the eikonal result and the jet and photon $p_{T}$ distributions we obtain using MCFM and hence the full $2 \rightarrow 3$ matrix elements, imposing a cut of $200 \mathrm{GeV}$ on the jet $p_{T}$. The ratio of the two photon $p_{T}$ distributions is shown in Fig. 8 for centre-of-mass energies of $14 \mathrm{TeV}$ and $40 \mathrm{TeV}$. At the lower collider energy we terminate the distribution at $p_{T} \sim 2 \mathrm{TeV}$ as we run into low statistics. In this plot we also include the ratio for the corresponding process obtained for a centre-of-mass energy of $40 \mathrm{TeV}$. Evidently the two results agree much better at $40 \mathrm{TeV}$, as the eikonal condition is better satisfied at higher energies.

Another effect we can study is the impact of the cut on the jet transverse momentum on the photon $p_{T}$ distribution. This is shown for $u \bar{u} \rightarrow A g Z$ in Fig. 9. We see that as expected increasing the cut decreases the cross section and also modifies the shape at low $p_{T}$.

\subsubsection{The $\gamma$ to $Z$ ratio}

The importance of the ratio of the $\gamma+$ jets to $Z+$ jets cross sections in new physics searches has been discussed in [17]. The ratio is useful to better calibrate the missing transverse energy plus jets background to SUSY production coming from $Z(\rightarrow$ neutrinos $)$ plus jets. Leading order results have been shown in [17] for the ratio of $\gamma$ to $Z$ for one or more jets. The ratio is known to be insensitive to NLO QCD corrections, and suffers less than the individual cross sections from scale and PDF uncertainties. The effect of virtual corrections on this ratio has been considered in [3]. The results are shown in Fig. 10. We note that virtual corrections increase the ratio at large $p_{T}$ because the $Z$ cross section is decreased more than the $\gamma$ cross section by the virtual DL. However, the inclusion of soft real radiation 

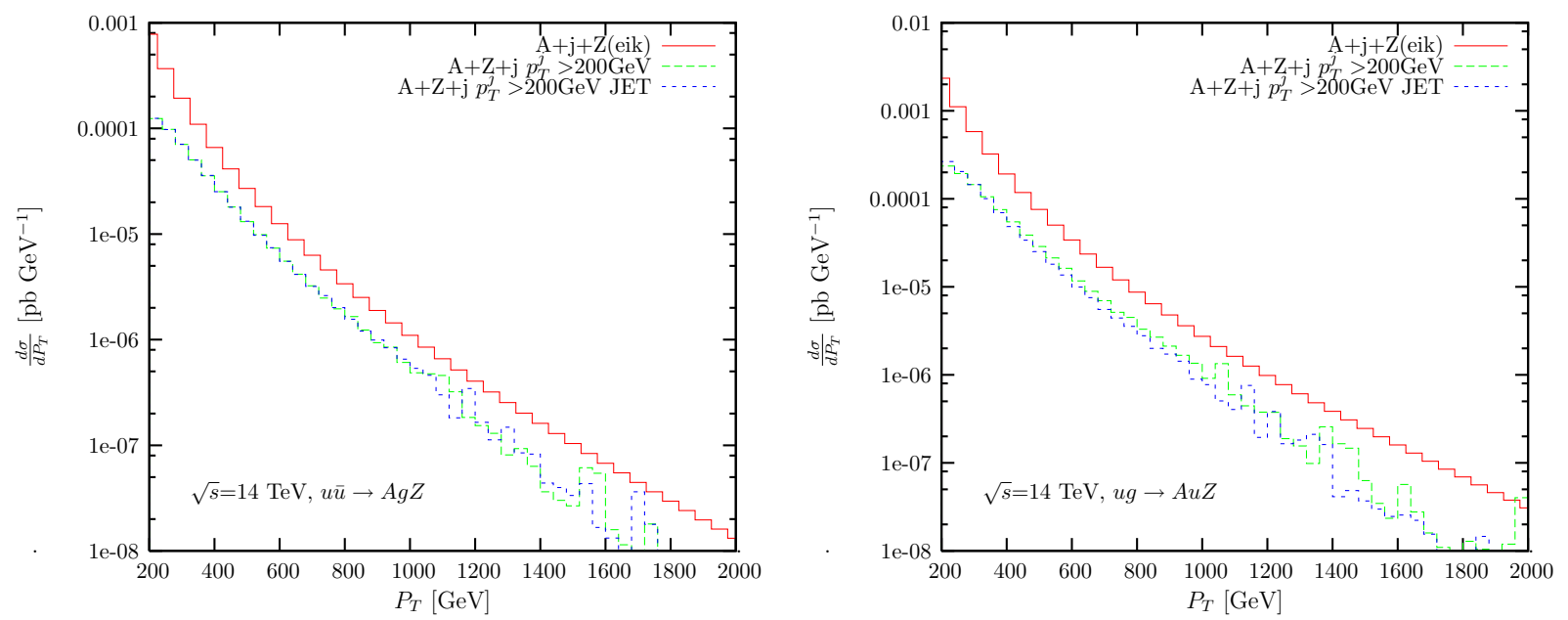

Figure 6: Photon transverse momentum distribution for $u \bar{u} \rightarrow A g Z$, for soft $Z$ (eikonal current) and using MCFM, with fixed scales.

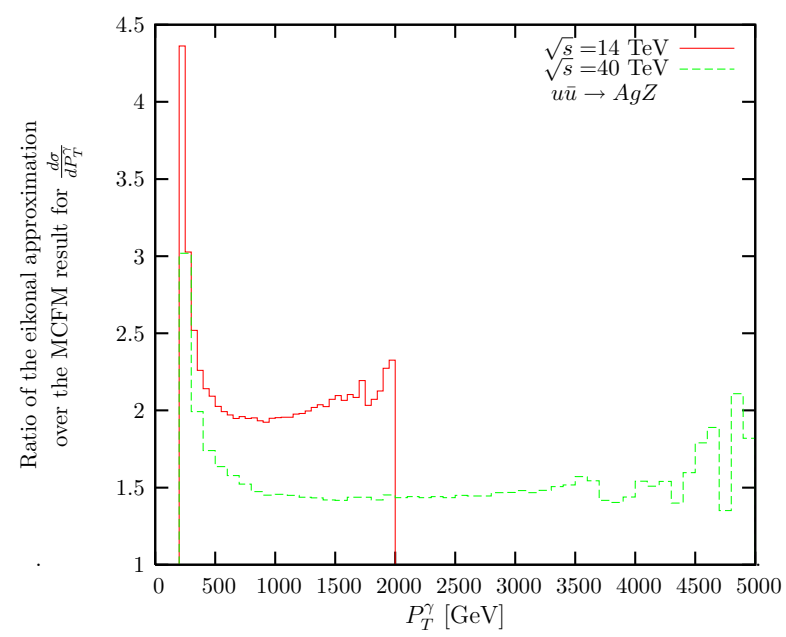

Figure 8: Ratio of the eikonal approximation result to the $2 \rightarrow 3$ MCFM result for the subprocess $u \bar{u} \rightarrow A g Z$.
Figure 7: Photon transverse momentum distribution for $u g \rightarrow A u Z$, for soft $Z$ (eikonal current) and using MCFM, with fixed scales.

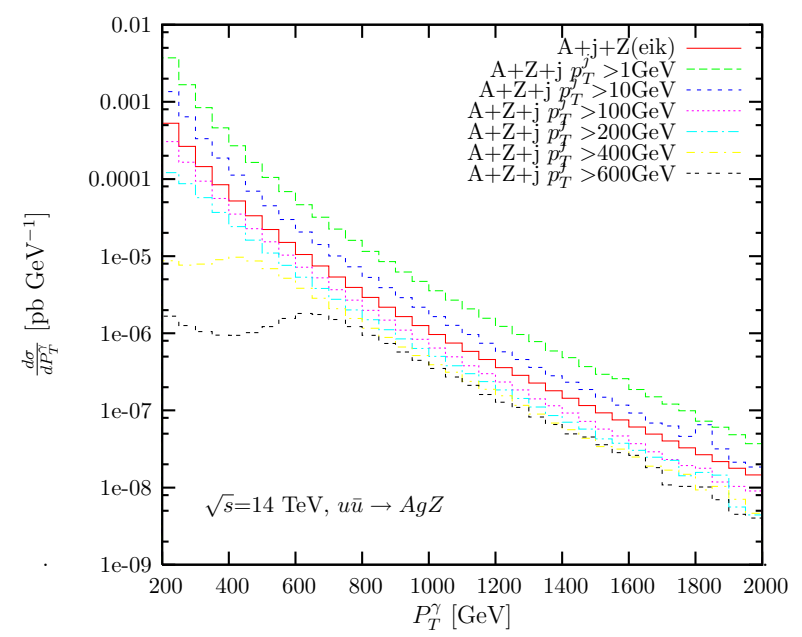

Figure 9: Effect of the jet $p_{T}$ cut on the photon transverse momentum distribution for subprocess $u \bar{u} \rightarrow A g Z$.

in the eikonal limit significantly shifts the result closer to the LO value. In practice, this of course depends on the amount of soft and collinear radiation that is allowed to escape detection. Setting cuts on the emitted bosons and their decay products reduces the effect of the real corrections leading to a result closer to the virtual result of [3]. A detailed study including cuts and experimental restrictions, which is beyond the scope of this work, would be needed to determine how rapidly this convergence occurs. 


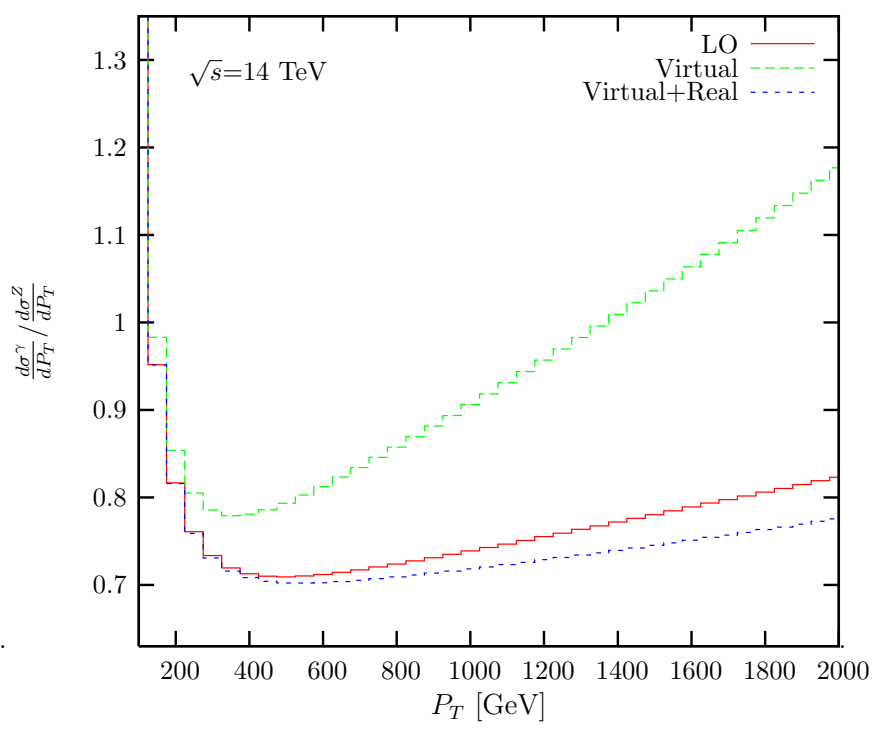

Figure 10: Ratio of $\gamma+$ jet to $Z+$ jet cross sections as a function of $p_{T}$ at LO, including both virtual corrections and real corrections at the DL level.

\section{Bloch-Nordsieck violations in other hard-scattering processes}

\subsection{QCD dijet production}

Electroweak corrections for dijet production have been studied in [4], [18] and recently in [19]. The virtual one-loop EW corrections $\left(\mathcal{O}\left(\alpha_{s}^{2} \alpha_{w}\right)\right)$ to the single jet inclusive cross section have first been studied in [4]. These are found to decrease the LO cross section $\left(\mathcal{O}\left(\alpha_{s}^{2}+\alpha_{s} \alpha_{w}+\alpha_{w}^{2}\right)\right)$ by up to $35 \%$ at very large jet energies $(\sim 4 \mathrm{TeV})$ at the LHC. It is noted in [4] that $W$ - and $Z$-bremsstrahlung effects might counterbalance the negative logs of the virtual corrections, as discussed in [20]. In this case the calculation of the $\mathcal{O}\left(\alpha_{s}^{2} \alpha_{w}\right)$ result is complicated by the presence of the LO purely electroweak processes increasing the number of Feynman diagrams, which lead to different possible interferences that must be taken into account. At tree level the contribution of $\mathcal{O}\left(\alpha_{s} \alpha_{w}+\alpha_{w}^{2}\right)$ terms is found in [4] to reach $15 \%$ of the QCD contribution at $4 \mathrm{TeV}$.

In this section we present a qualitative discussion focused on the different subprocesses contributing to $2 \rightarrow 2$ QCD scattering and the corresponding EW corrections at the DL level, examining which subprocesses exhibit BN violations and their origin, based on a Feynman diagram approach. However in order to simplify the discussion we ignore the pure LO EW contributions, so that the $\mathcal{O}\left(\alpha_{s}^{2} \alpha_{w}\right)$ corrections come from the interference of the $\operatorname{LO} \mathcal{O}\left(\alpha_{s}\right)$ matrix element with the $\operatorname{NLO} \mathcal{O}\left(\alpha_{s} \alpha_{w}\right)$ matrix element. To identify which processes exhibit $\mathrm{BN}$ violations, we need to focus only on virtual and real $W$ corrections as the $Z$ corrections always cancel, as shown by the form of Eqs. (2.6) and (2.7). Here we briefly consider the subprocesses as organised in [4]. The classification is based on the initial state parton combinations which determine the PDF weight. The examples given are for the first generation of quarks, but the same arguments hold for the second generation. In what follows, the CKM matrix is taken to be diagonal. Quantitative results will be 
presented in another study. We note that consideration of the full real corrections involves calculating processes such as $W / Z+2$ jets production.

- $\underline{g g \rightarrow g g}$ This subprocess receives no real or virtual corrections at $\mathcal{O}\left(\alpha_{s}^{2} \alpha_{w}\right)$.

- $g g \rightarrow q \bar{q}$ The corrections consist of real and virtual $W$ emission from the final-state legs. As we are summing over all quark flavours in the final state the divergences cancel. This can be inferred from the form of Eqs. (2.6) and (2.7): both the real and virtual corrections are proportional to $M_{0}^{g g d \bar{d}} M_{0}^{* g g u \bar{u}}$ and therefore the cancellation of Sudakov logs is exact.

- $\underline{q \bar{q} \rightarrow g g}$ As the initial state is fixed to be a same flavour quark-antiquark pair, real $W$ emission is not allowed in this subprocess. Therefore the DL coming from the exchange of a soft $W$ boson between the initial state quarks, as shown in Fig. 11, remain uncancelled. We note that processes of type $u \bar{d} \rightarrow W^{+} g g$ are considered separately below.
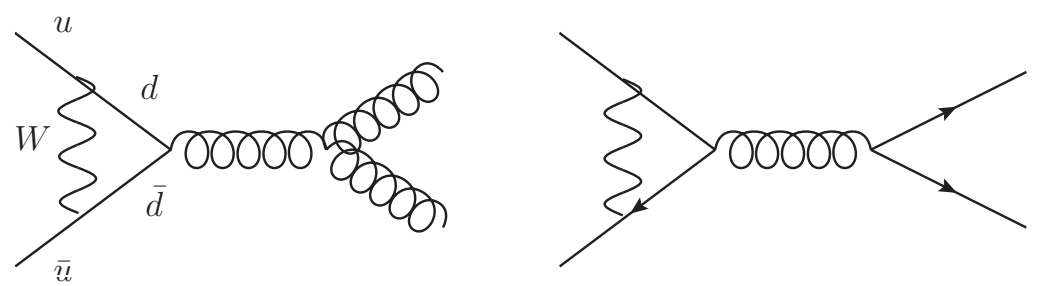

Figure 11: Virtual correction diagrams for jet production from initial state $u \bar{u}$.

- $\underline{q g \rightarrow q g(\bar{q} g \rightarrow \bar{q} g)}$ Real emission takes place from the initial- and final-state quark legs. Real and virtual corrections are both proportional to $M_{0}^{u g u g} M_{0}^{* d g d g}$ leading to exact cancellation between real and virtual corrections, and therefore no $\mathrm{BN}$ violation is expected.

- $\underline{q q \rightarrow q q(\bar{q} \bar{q} \rightarrow \bar{q} \bar{q})} \mathrm{BN}$ violating logs are present. This can be seen by noting that for the real corrections we obtain the interference between the diagram where $W$ is emitted from $q\left(p_{1}\right)$ and that where $W$ is emitted from $q\left(p_{2}\right)$, leading to a term proportional to $\log ^{2}\left(2 p_{1} p_{2} / M_{W}^{2}\right)$. In the virtual corrections no Feynman diagram can be drawn with $W$ exchanged between the initial state legs and therefore the real DL remain uncancelled. The remaining $t$ and $u$ double logs cancel. (This subprocess is in general complicated by the presence of the two diagrams at LO ( $u-$ and $t$-channel), with the real corrections not being proportional to $\left|M_{L O}\right|^{2}$.)

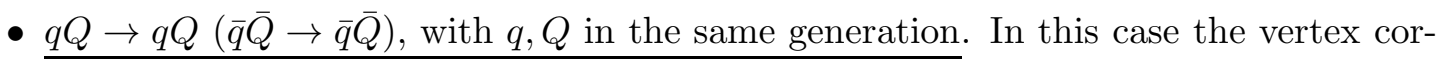
rection diagrams exactly cancel the interference of diagrams with emission from the same flavour fermion line. However as we have quarks of the same generation a set of box diagrams is also allowed. These are shown in Fig. 12. There is no corresponding set of real emission diagrams to cancel the divergences from these diagrams, as a $W^{+}$ 
is emitted from one flavour leg but a $W^{-}$from the other, and we therefore expect $\mathrm{BN}$ violating logs.
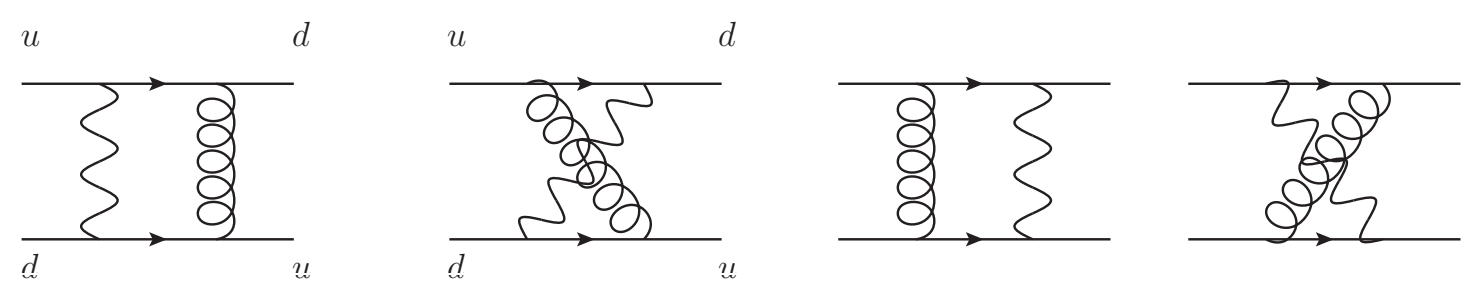

Figure 12: Box diagram corrections for $q Q \rightarrow q Q$ scattering.

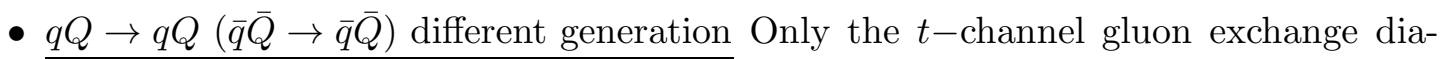
gram exists at LO. The virtual corrections consist only of the vertex corrections diagrams with the divergences exactly cancelling those of the real emission diagrams. Interference occurs only between the diagrams where emission comes from the same flavour initial and final legs. No BN violation is therefore expected from this subprocess.

- $q \bar{Q} \rightarrow q \bar{Q}$ different generation The same conclusions as in the previous subprocess apply, and no BN violation is expected.

- $\underline{q} \rightarrow q \bar{Q}$ same generation In this case we note that for initial state $q \bar{Q}$ at $\mathcal{O}\left(\alpha_{s}^{2} \alpha_{w}\right)$ the real corrections involve processes of type $u \bar{d} \rightarrow W^{+} q \bar{q}$, with $q$ of any flavour and $u \bar{d} \rightarrow W^{+} g g$. Moreover in the virtual corrections we have box diagrams which can lead to a different generation pair in the final state. The divergences from the interference of the $s$-channel diagrams where a $W$ is emitted from the two initial state legs remain uncancelled, as in the virtual corrections a $W$ cannot be exchanged between the initial-state quark pair. The set of diagrams that lead to uncancelled divergences is shown in Fig. 13 for $u \bar{d}$ scattering.

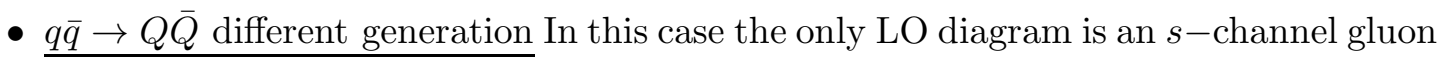
exchange. The virtual corrections include a diagram where a $W$ boson is exchanged between the $q \bar{q}$ pair, shown in Fig. 11 for $u \bar{u}$. This leads to a DL which cannot be cancelled by the real corrections.

- $\underline{q \bar{q} \rightarrow q \bar{q}}$ The same loop diagram as in the previous case leads to BN violating DL.

- $\underline{q \bar{q} \rightarrow Q \bar{Q}}$ same generation In addition to the vertex correction $s$-channel diagram of the previous two cases, in this case two $t$-channel box diagrams shown in Fig. 14 are also allowed. These loop diagrams also lead to uncancelled DL.

The importance of the BN violating DL can be estimated by considering Eqs. (2.6) and (2.7), and also the relative importance of the various subprocesses for the dijet cross section at the LHC. This is shown in Fig. 15 with the cross section decomposed into subprocesses based on the initial partonic combination. A similar decomposition is shown in Table 1 of 

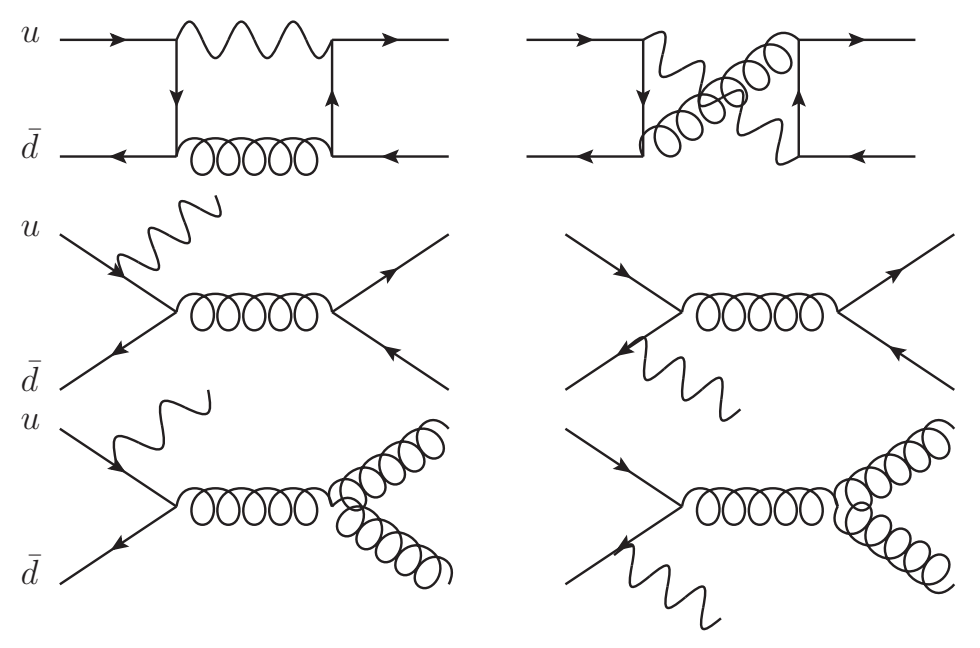

Figure 13: Sample diagrams for initial state $u \bar{d}$ scattering.
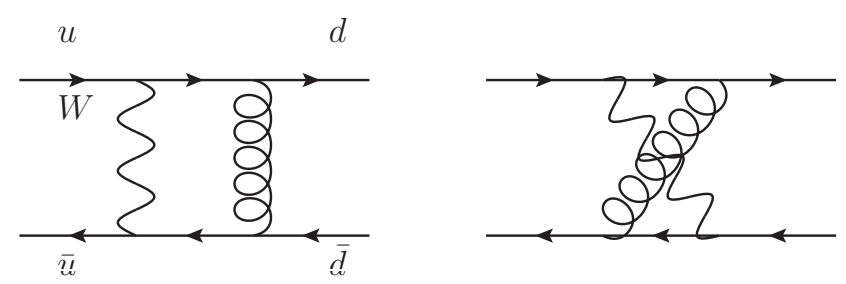

Figure 14: Sample diagrams leading to BN violating logs for initial-state $u \bar{u}$ scattering.

Ref. [4], where the contribution to the total EW corrections is decomposed into different subprocesses. To quantify the exact combined effect of real and virtual EW corrections would require a full calculation of both corrections.

\subsection{Electroweak processes}

\subsection{1 $\mathrm{W}+\mathrm{Z}$ production}

For comparison with the QCD case, we also consider BN violating effects in a purely electroweak process, i.e. $W+Z$ hadronic production. Once again we will not present any detailed numerical results, rather simply a qualitative discussion. The virtual corrections for this process have been studied in [21] where analytic results obtained using Eq. (2.6) are presented in the high-energy limit. In this limit longitudinal gauge bosons can be replaced by the corresponding would-be Goldstone bosons. The virtual corrections are found in [21] to decrease the cross section by up to $20 \%$ at $p_{T}=500 \mathrm{GeV}$. The calculation of the real corrections involves considering the processes $q \bar{q} \rightarrow W^{+} Z W^{-}$and $q \bar{Q} \rightarrow W^{+} Z Z$. We have obtained the partonic results for these processes, but the expressions are long and complicated due to the presence of several diagrams. Note that at this NLO-EW order new partonic PDF combinations are allowed that are not present at leading order (e.g. $u \bar{u}$ scattering does not contribute at leading order but produces the $W^{+} Z W^{-}$final state at 


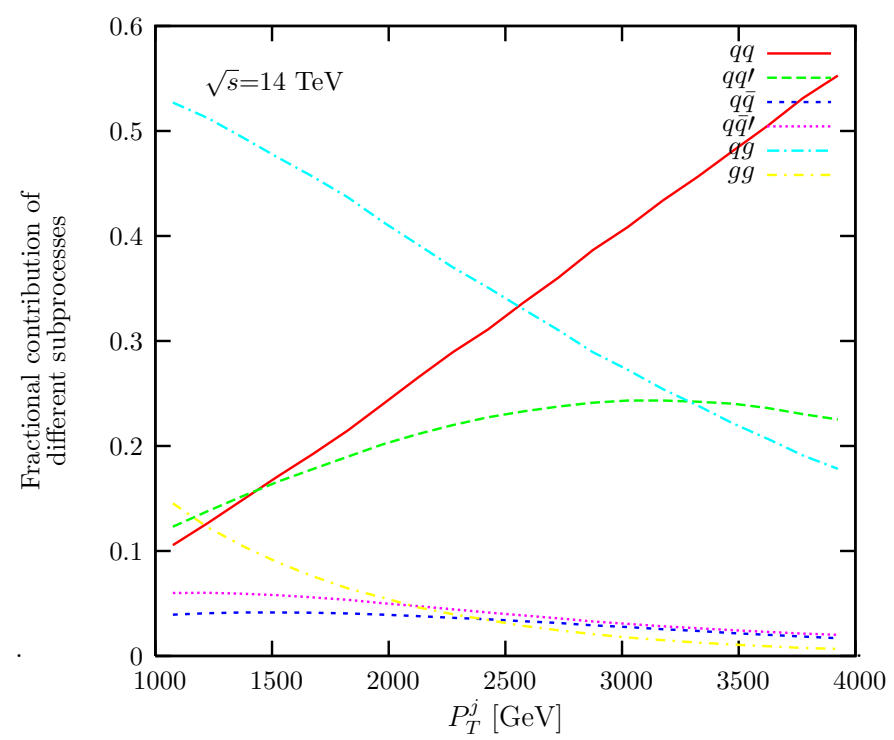

Figure 15: Fractional contributions of different subprocesses as a function of the jet transverse momentum at LO.

NLO). We therefore expect BN violating logs that will not cancel even after the addition of the virtual corrections, which are weighted by a different PDF (LO) combination.

\subsection{2 $\mathrm{W}+\mathrm{H}$ production}

The full $\mathcal{O}(\alpha)$ virtual EW and real photon emission corrections to Higgs production in association with a $W$ boson have been studied in [22]. Here we are only interested in the DL corrections, which can be extracted and studied analytically. At LO the process $u \bar{d} \rightarrow W^{+} H$ has only one Feynman diagram (in the diagonal CKM matrix approximation). The relevant virtual correction diagrams are shown in [22]. For the real corrections the processes to be considered are $u \bar{d} \rightarrow W^{+} H Z$ and the new channel $q \bar{q} \rightarrow W^{+} H W^{-}$. For illustration, we consider the matrix element squared for the new channel $u\left(p_{1}\right) \bar{u}\left(p_{2}\right) \rightarrow$ $W^{+}\left(p_{3}\right) H\left(p_{4}\right) W^{-}$which factorises in the high-energy (soft $W^{-}$) limit:

$$
\begin{aligned}
|M|^{2} & =\frac{\alpha_{w}}{24 \pi c_{w}^{2} s_{w}^{2}}\left(\left(3-2 s_{w}^{2}\right) \log ^{2}\left(\frac{2 p_{2} p_{4}}{M_{W}^{2}}\right)+\left(3-4 s_{w}^{2}\right) \log ^{2}\left(\frac{2 p_{2} p_{3}}{M_{W}^{2}}\right)\right. \\
& \left.-\frac{\left(9-18 s_{w}^{2}-8 s_{w}^{4}\right)}{6} \log ^{2}\left(\frac{2 p_{3} p_{4}}{M_{W}^{2}}\right)\right)\left|M_{\mathrm{LO}}\right|^{2},
\end{aligned}
$$

where the intermediate photon contribution is also included. This contribution cannot be cancelled by the virtual corrections, since $u \bar{u}$ scattering does not contribute at leading order. The corresponding result for $u\left(p_{1}\right) \bar{d}\left(p_{2}\right) \rightarrow W^{+}\left(p_{3}\right) H\left(p_{4}\right) Z$ in the same limit is

$$
\begin{aligned}
|M|^{2} & =\frac{\alpha_{w}}{72 \pi c_{w}^{2} s_{w}^{2}}\left(-\left(3-2 s_{w}^{2}\right)\left(3-4 s_{w}^{2}\right) \log ^{2}\left(\frac{2 p_{1} p_{2}}{M_{Z}^{2}}\right)+3\left(3-4 s_{w}^{2}\right)\left(1-s_{w}^{2}\right) \log ^{2}\left(\frac{2 p_{1} p_{3}}{M_{Z}^{2}}\right)\right. \\
& +3\left(3-4 s_{w}^{2}\right) \log ^{2}\left(\frac{2 p_{1} p_{4}}{M_{Z}^{2}}\right)+3\left(3-2 s_{w}^{2}\right) \log ^{2}\left(\frac{2 p_{2} p_{3}}{M_{Z}^{2}}\right)+3\left(3-2 s_{w}^{2}\right)\left(1-2 s_{w}^{2}\right) \log ^{2}\left(\frac{2 p_{2} p_{4}}{M_{Z}^{2}}\right)
\end{aligned}
$$




$$
\left.-9\left(1-2 s_{w}^{2}\right) \log ^{2}\left(\frac{2 p_{3} p_{4}}{M_{Z}^{2}}\right)\right)\left|M_{\mathrm{LO}}\right|^{2}
$$

where we have not used the high-energy approximation $p_{3} p_{4}=p_{1} p_{2}$ in order to distinguish between logs from the interference of different diagrams. In this form it is straightforward to identify how the different prefactors arise from the different $Z$ couplings to $u, d, W$ and Higgs. The DL in this expression exactly cancel if we consider the 1-loop diagrams with virtual $Z$ exchange.

\section{Conclusions}

We have studied the effect of electroweak corrections on a range of $2 \rightarrow 2$ scattering processes at the LHC. Existing studies of electroweak corrections generally only take account of the virtual contributions, as the real emission of EW bosons leads to different final states in the detectors. Nevertheless when sufficiently inclusive measurements are considered, the emission of soft electroweak bosons can significantly counterbalance the effect of large negative Sudakov logs from the virtual corrections. Even though the cancellation is never exact because of violations of the Bloch-Nordsieck theorem in electroweak corrections, we have found that the cancellation can be numerically significant. We have only studied the corrections at the DL level, but we can gauge the effect of real radiation, and this has been done at the hadronic level for $\gamma / Z+$ jet production, showing that the real radiation effects counterbalance the virtual corrections to a large extent.

We have also studied a sample of other $2 \rightarrow 2$ QCD and EW processes, identifying potentially large $\mathrm{BN}$ violating contributions by considering the relevant Feynman diagrams. For these and other cases, detailed numerical studies going beyond the DL approximation would be needed to quantify the exact impact of the BN violating contributions on hadronic cross sections and these would need to include the effect of phase space constraints on the emitted $W, Z$ bosons and their decay products.

\section{Acknowledgments}

E.V. acknowledges financial support from the UK Science and Technology Facilities Council.

\section{References}

[1] J. H. Kuhn, A. Kulesza, S. Pozzorini and M. Schulze, Phys. Lett. B 609, 277 (2005) [hep-ph/0408308].

[2] J. H. Kuhn, A. Kulesza, S. Pozzorini and M. Schulze, Nucl. Phys. B 727, 368 (2005) [hep-ph/0507178].

[3] J. H. Kuhn, A. Kulesza, S. Pozzorini and M. Schulze, JHEP 0603, 059 (2006) [hep-ph/0508253].

[4] S. Moretti, M. R. Nolten and D. A. Ross, Nucl. Phys. B 759, 50 (2006) [hep-ph/0606201]. 
[5] S. Moretti, M. R. Nolten and D. A. Ross, Phys. Lett. B 639, 513 (2006) [Erratum-ibid. B 660, 607 (2008)] [hep-ph/0603083].

[6] J. H. Kuhn, A. Scharf and P. Uwer, Eur. Phys. J. C 45, 139 (2006) [hep-ph/0508092].

[7] J. H. Kuhn, A. Scharf and P. Uwer, Phys. Rev. D 82, 013007 (2010) [arXiv:0909.0059 [hep-ph]].

[8] M. Ciafaloni, P. Ciafaloni and D. Comelli, Phys. Rev. Lett. 84, 4810 (2000) [hep-ph/0001142].

[9] M. Ciafaloni, P. Ciafaloni and D. Comelli, Nucl. Phys. B 589, 359 (2000) [hep-ph/0004071].

[10] M. Ciafaloni, P. Ciafaloni and D. Comelli, Phys. Lett. B 501, 216 (2001) [hep-ph/0007096].

[11] M. Ciafaloni, P. Ciafaloni and D. Comelli, Phys. Rev. Lett. 87, 211802 (2001) [hep-ph/0103315].

[12] U. Baur, Phys. Rev. D 75, 013005 (2007) [hep-ph/0611241].

[13] G. Bell, J. H. Kuhn and J. Rittinger, Eur. Phys. J. C 70, 659 (2010) [arXiv:1004.4117 [hep-ph]].

[14] A. Denner and S. Pozzorini, Eur. Phys. J. C 18, 461 (2001) [hep-ph/0010201].

[15] A. D. Martin, W. J. Stirling, R. S. Thorne and G. Watt, Eur. Phys. J. C 63, 189 (2009) [arXiv:0901.0002 [hep-ph]].

[16] J. M. Campbell and R. K. Ellis, http://mcfm.fnal.gov/.

[17] S. Ask, M. A. Parker, T. Sandoval, M. E. Shea and W. J. Stirling, JHEP 1110, 058 (2011) [arXiv:1107.2803 [hep-ph]].

[18] A. Scharf, arXiv:0910.0223 [hep-ph].

[19] S. Dittmaier, A. Huss and C. Speckner, arXiv:1210.0438 [hep-ph].

[20] P. Ciafaloni and D. Comelli, JHEP 0609, 055 (2006) [hep-ph/0604070].

[21] E. Accomando, A. Denner and S. Pozzorini, Phys. Rev. D 65, 073003 (2002) [hep-ph/0110114].

[22] M. L. Ciccolini, S. Dittmaier and M. Kramer, Phys. Rev. D 68, 073003 (2003) [hep-ph/0306234]. 